\title{
Modelling the Influence of Personality and Culture on Affect and Enjoyment in Multimedia
}

\author{
Anonymized Submission
}

\begin{abstract}
Affect is evoked through an intricate relationship between the characteristics of stimuli, individuals, and systems of perception. While affect is widely researched, few studies consider the combination of multimedia system characteristics and human factors together. As such, this paper explores the influence of personality (Five-Factor Model) and cultural traits (Hofstede Model) on the intensity of multimedia-evoked positive and negative affects (emotions). A set of 144 video sequences (from 12 short movie clips) were evaluated by 114 participants from a cross-cultural population, producing 1232 ratings. On this data, three multilevel regression models are compared: a baseline model that only considers system factors; an extended model that includes personality and culture; and an optimistic model in which each participant is modelled. An analysis shows that personal and cultural traits represent $5.6 \%$ of the variance in positive affect and $13.6 \%$ of the variance in negative affect. In addition, the affect-enjoyment correlation varied across the clips. This suggests that personality and culture play a key role in predicting the intensity of negative affect and whether or not it is enjoyed, but a more sophisticated set of predictors is needed to model positive affect with the same efficacy.
\end{abstract}

\section{INTRODUCTION}

Every day we witness several organizations put forward their missions in the form of ad campaigns. While most of these ads fail to attract our attention, some of them leave a lasting impression in our minds. Take the example of the campaign by Dove (a beauty product range by Unilever [1]), which was listed as one of most unforgettable ad campaigns of 2013 [2] or the United Nations Foundation's campaign for World Humanitarian Day [3]. The huge success of such ad campaigns is attributed to how story-telling components are shaped into emotion-evoking communication, structured to stimulate action. Perceptions of warmth and competence shape our judgements of people and organizations, and when perceived together they cause active behavioral responses from the viewers [4].

Ad campaigns are but one specific scenario which illustrate the importance and challenge of modeling multimedia-evoked emotions. The problem is not just limited to content- or genrebased analysis of multimedia. This is because a video which arouses a positive emotion in one person might arouse a negative emotion in the other (depending on the nature of content and users' cultural and psychophysical frameworks which influence their perception) [5]. Therefore it involves understanding the subjective nature of emotions and how crucial a role human factors play in modeling experience of affect (emotion), thereby addressing users' needs for emotionsensitive video retrieval [6].
In this work, we attempt to understand how personality [7] and culture [8] influence users' experience of affect and enjoyment in multimedia. Specifically, the following research questions are posed:

RQ 1. Can a model based on multimedia system characteristics and human factors (i.e., personality and culture) predict the intensity of affect (both positive and negative) and enjoyment?

RQ 2. Which system characteristics and human factors influence the experience of affect and enjoyment the most?

RQ 3. What is the relationship between experience of affect (both positive and negative) and enjoyment across stimuli?

By investigating how different dimensions of these human factors modulate users' experience of affect and enjoyment, we intend to provide some initial findings for multimedia content creators to achieve maximal user satisfaction with respect to the contents they create and deliver to diverse users.

\section{RELATED WORK}

There is a broad range of research that aims to predict affective responses to multimedia (see [9] for a thorough review). Some focus on distilling the influence of specific cinematographic theories [10], types of segment and shot [11], the use of colour [12] and connotative space [13]. Others focus on modelling these different audio-visual features to predict emotions [14]. Such research on modeling emotional response in videos often takes into account the facial expressions of viewers [15] and a range of complementary sensors (e.g., heart rate) to help measure the evoked emotions [16], [17]. However, the extent to which physiological responses capture the subjective intensity of affects (which vary as a consequence of users' innate psychology) is unclear.

A consequence of this is that such studies implicitly assume that, given a video, the affect experienced by different users will be more or less the same. This is equally the case with affective video datasets (e.g., [18], [19], [20], [21], [22]). However prior research shows that individual differences can lead to varied experiences [5]. To illustrate this, evidence reveals a complex relationship between affective video tagging and physiological signals [6], [23], [24]. As such, it is important to consider the subjective nature of affective perception.

These individual differences are subtle, owing to the complexity associated with the different dimensions of individuals. These differences occur both at a micro-cosmic level (i.e., specific to the individual) and at a macro-cosmic level (i.e., 
cultural influences). While the micro-cosmic traits can be analysed with personality (a series of "internal properties" that relate to overt behaviours [7]), the macro-cosmic traits can be captured using cultural dimensions (representing "the collective programming of the mind distinguishing the members of one [nation] or category of people from others" which subsequently leads to a "broad tendency [for members of a group] to prefer certain states of affairs over others" [25]).

These two human factors, namely personality and culture, are shown to reliably capture individual differences in multiple domains like language [26], intonation of voice while speaking [27], kind of photos one likes [28], type of people one befriends [29] etc. (see [30] for a thorough review). Other examples include preference of genre for language learning in different cultures [31] and the respective cultural acceptance of some movie content [32] etc. Due to the consistency shown between these human factors and user behaviors, we use them to study how they influence users' experience of affect and enjoyment in multimedia, which to the best of our knowledge is an unexplored research topic.

\section{Methodology}

\section{A. Video Dataset}

This study is the first application of the CP-QAE-I dataset (available from [removed for review purposes]). This dataset contains 144 video sequences in MP4 format and each sequence varies according to several parameters. There is 1 nominal parameter: content. This consists of 12 short clips from popular movies which have been purposively selected to cover different affective categories [22]. Movie clips of different valence, and which had least variation in ratings on arousal [22], were taken for this study to minimize any content-based biases (a list of the clips along with mean affect scores are seen in Table II). The content parameter also varies in cinematographic techniques and the technologies that were used during the original production of the movies. Additionally, there are 3 ordinal parameters: Bit-Rate $(384 \mathrm{~kb} / \mathrm{s}$ and $768 \mathrm{~kb} / \mathrm{s})$; Frame Size (480p and 720p); and Frame Rate (5fps, 15fps and 25fps). Thus, in the dataset, there are 12 quality conditions (resulting from the $3 * 2 * 2$ settings of FrameRate, Frame-Size (resolution) and Bit-Rate variables) and 12 emotion conditions (resulting from $2 * 6$ primary emotions [22]), thus resulting in a set of 144 'video sequences' (12*12). Each video sequence has a length between 1 and 3 minutes.

\section{B. Sample Size}

A power analysis was conducted in $\mathrm{G}^{*}$ Power 3 to calculate the required sample size based on the use of the $F$-statistic and repeated measures. Using conventional error probabilities $(\alpha=.05, \beta=0.2)$ and assuming medium effects $(f=0.39)$ with correlation $(r=0.8)$, a minimum size of 64 was suggested.

\section{Participants}

The participants were 114 university students drawn from the two institutions the authors are affiliated with. Exactly
TABLE I

SAMPLE DESCRIPTIVES

\begin{tabular}{lrrrr}
\hline Human Factors & Min & Max & $\bar{x}$ & $\sigma$ \\
\hline Extroversion & 2 & 9 & 5.54 & 1.689 \\
Agreeableness & 3 & 10 & 7.22 & 1.533 \\
Conscientiousness & 2 & 10 & 6.55 & 1.523 \\
Neuroticism & 2 & 10 & 5.62 & 1.716 \\
Openess & 4 & 10 & 6.75 & 1.424 \\
Power Distance & -155 & 140 & -35.96 & 53.219 \\
Individualism & -140 & 140 & 18.73 & 50.619 \\
Masculinity & -140 & 105 & -1.23 & 53.483 \\
Uncertainty Avoidance & -120 & 130 & 44.61 & 47.182 \\
Pragmatism & -130 & 155 & 16.84 & 58.090 \\
Indulgence & -220 & 185 & -16.97 & 65.522 \\
\hline
\end{tabular}

TABLE II

Marginal Means of PERCEIVED RESPONSES (AFFECT AND ENJOYMENT) ON CLIPS, AFTER FIXING THE CO-VARIATES

\begin{tabular}{lrrr}
\hline MovieClip & +ve Affect & -ve Affect & Enjoyment \\
\hline A_FISH_CALLED_WANDA & 0.184 & -0.536 & -0.037 \\
AMERICAN_HISTORY_X & -0.397 & 0.756 & -0.607 \\
CHILDS_PLAY_II & -0.231 & 0.698 & -0.158 \\
COPYCAT & -0.33 & 0.418 & -0.315 \\
DEAD_POETS_SOCIETY_1 & -0.331 & 0.341 & -0.504 \\
DEAD_POETS_SOCIETY_2 & 1.053 & -0.553 & 0.725 \\
FOREST_GUMP & 0.992 & -0.523 & 0.656 \\
SE7EN_1 & -0.346 & 0.248 & 0.42 \\
SE7EN_3 & -0.431 & 0.03 & -0.306 \\
SOMETHING_ABOUT_MARY & 0.468 & -0.72 & 0.471 \\
THE_PROFESSIONAL & -0.194 & 0.216 & 0.254 \\
TRAINSPOTTING & -0.477 & -0.389 & -0.654 \\
\hline
\end{tabular}

Based on estimated marginal means of a mixed-effects regression model. Covariates in the model are evaluated at the following values: EXTRAVERSION $=5.42 ;$ AGREEABLENESS $=7.45 ;$ CONSCIENTIOUSNESS $=6.59 ;$ NEUROTICISM $=5.67 ;$ OPENNESS $=6.77 ;$ POWER DISTANCE $=-34.29 ;$ INDIVIDUALISM $=22.44 ;$ MASCULINITY $=-6.73$; UNCERTAINTY AVOIDANCE $=40.83 ;$ PRAGMATISM $=22.82 ;$ INDULGENCE $=-11.60$.

$50 \%$ of the sample was drawn from each institution. In terms of nationality, there were: 43 British, 22 Indian, 16 Chinese, 15 Singaporean and 18 from different nationalities. The proportion of female participants was $28.9 \%$ and the average age was 23.9 years $(\sigma=3.68)$. Additional descriptives in terms of personality and cultural traits are shown above in Table I.

\section{Procedure}

This study applied a lab-based subjective testing approach. A set of video sequences were hosted on web server locally at each institution involved in the study. Participants were asked to access an online questionnaire from the corresponding local network (to avoid any latency issues over the Internet). They then watched several video sequences and rated the experience of affect and their enjoyment of each sequence by completing questions immediately after viewing each. Informed consent and anonymity were assured at every stage of the study.

Participants started the survey by answering the VSM-2013 [33] and the BFI-10 [34] questionnaires to report cultural and personality traits respectively. Then each participant was 


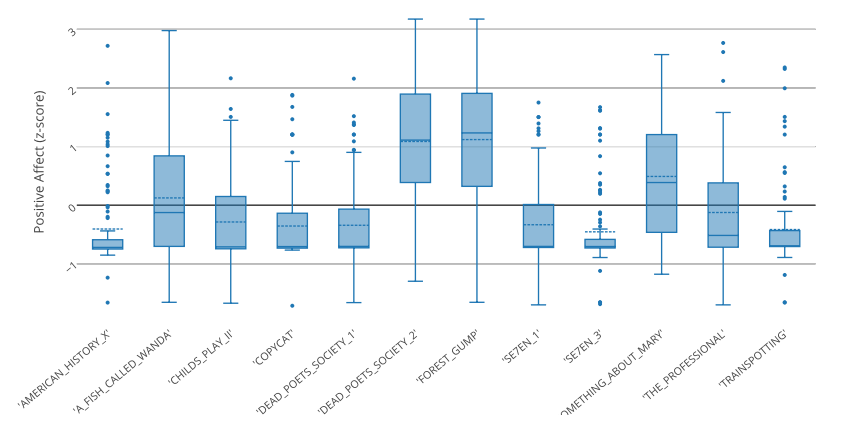

Fig. 1. Relative mean levels of positive affect that participants rated each video sequence.

shown 12 video sequences under assessment. Each participant was expected to rate all 12 video sequences. Of the 114 participants, $73.7 \%$ rated all 12 . The minimum number of videos rated was 3 , however the average was $10.8(\sigma=2.56)$. In total, 1232 ratings were recorded ( $90 \%$ of the maximum possible).

\section{E. Measures}

1) Positive and Negative Affect: measured using Differential Emotions Scale [35]. This includes 16 sets of of emotional adjectives. Each set was linked to a 5-point Likert-type item so participants could indicate the extent they felt the corresponding emotion. Aggregate scores for positive affect (i.e., joy, warmth, love, calm, etc.) and negative affect (i.e., anger, fear, anxiety, sadness, etc.) were computed by a summation of the respective items. (Descriptive Statistics on ratings are shown in Figures 1,2)

2) Enjoyment: measured using a single 5-point Guttmantype scale, where participants indicated how much they enjoyed the video sequence. A score of 1 indicates "no" enjoyment while that of 5 indicates "high" enjoyment. (Descriptive Statistics on ratings are shown in Figure 3)

3) Culture: measured using the VSM-2013 questionnaire [33] according to the following dimensions: power distance (PDI); individualism (IDV); uncertainty avoidance (UAI); masculinity (MAS); pragmatism (PRG); and indulgence (IVR).

4) Personality: measured using the BFI-10 [34] questionnaire, according to the FFM [36], measuring: openness (Ope); conscientiousness (Con); Extroversion (Ext); Agreeableness (Agr); Neuroticism (Neu).

\section{RESULTS AND DisCUSSION}

In this section, we show results from experiments conducted to answer the research questions [RQ1,RQ2 and RQ3] raised at the outset of the paper. Sections IV-A and IV-B deals with RQ1 and RQ2, and Section IV-C deals with RQ3. Analysis was conducted in PASW 20.0 for Windows. Mixed linear regression was used to account for repeated measures. The parameters in each model were estimated together using the restricted maximum-likelihood method.

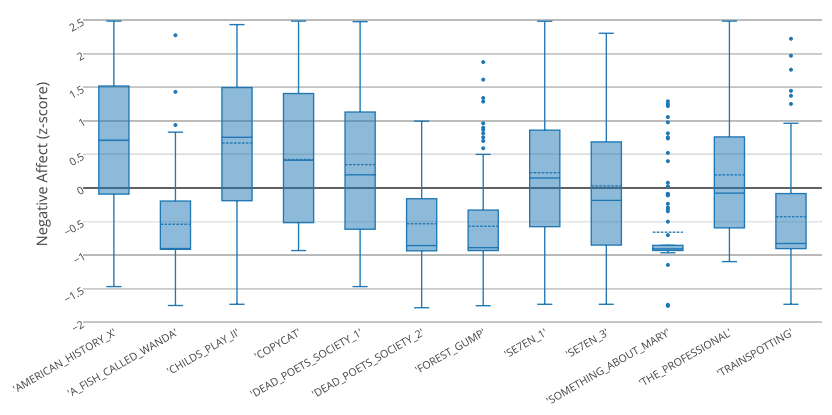

Fig. 2. Relative mean levels of negative affect that participants rated each video sequence.

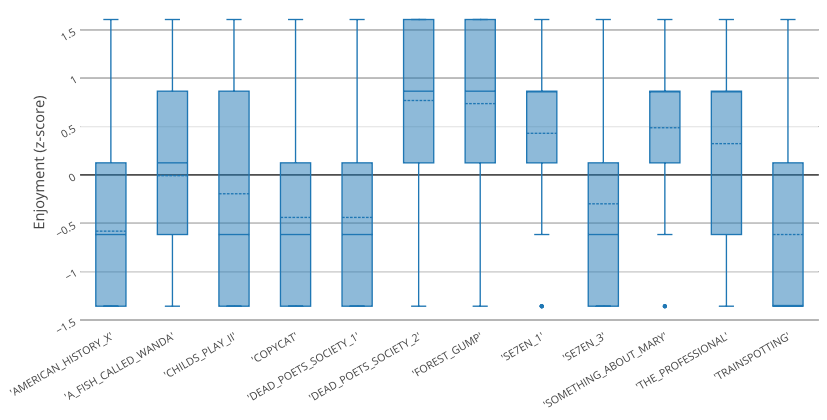

Fig. 3. Relative mean levels of Enjoyment that participants rated each video sequence.

\section{A. Models for Experience of Affect and Enjoyment}

We build three computational models (namely baseline, extended and optimistic) to investigate the influence of system factors (namely Bit-Rate, Frame-Rate and Frame-Size) and human factors (namely the five personality factors and six culture factors) on the experience of affect and enjoyment. Each of them along with corresponding findings will be described in this section. Then a comparison between the three models will be presented to address the three questions we pose in this paper.

a) Baseline Model: The baseline model only considers system factors. In the context of the CP-QAE-I video dataset, there are 12 variations of the system factors which were varied: Frame-Rate (3 conditions); Frame Size ( 2 conditions); and Bit-Rate (2 conditions). Other system factors such as file format and delivery protocol were held constant as part of the experimental setup. Due to expected interactions between these conditions (e.g., an attempt to minimise bit-rate while maximising Frame-Rate and Frame-Size would likely create artefacts) these were modelled as factorial interactions. In addition, the movie clip itself is included as a parameter to reflect differences in cinematographic technologies and techniques used to create the movies, along with the nature of the content. This was modelled as a main effect.

The results can be seen in Table III. The movie clip itself 
TABLE III

The Baseline FiXed-Effect Multilevel Linear Regression Model Predicting Three Dependent Variables

\begin{tabular}{|c|c|c|c|c|c|c|c|c|c|c|}
\hline \multirow[b]{2}{*}{ Parameter } & \multirow[b]{2}{*}{$d f_{\text {num }}$} & \multicolumn{3}{|c|}{ Positive Affect } & \multicolumn{3}{|c|}{ Negative Affect } & \multicolumn{3}{|c|}{ Enjoyment } \\
\hline & & $d f_{d e n}$ & $F$ & $\bar{p}$ & $d f_{d e n}$ & $F$ & $\bar{p}$ & $d f_{d e n}$ & $F$ & $p$ \\
\hline Movie C & 11 & 156.009 & 25.315 & .000 & 44.643 & 33.932 & .000 & 177.09 & 40.14 & .000 \\
\hline Frame Rate (F & 2 & 803.739 & .321 & .725 & 710.192 & .056 & .946 & 1131.23 & 5.173 & 0.006 \\
\hline Frame Size (FS) & 1 & 809.889 & .006 & .939 & 729.398 & 3.298 & .070 & 1146.39 & 2.846 & 0.092 \\
\hline Bit-Rate (BR) & 1 & 816.675 & 1.724 & .190 & 714.909 & .304 & .582 & 1139.69 & 0.474 & 0.491 \\
\hline
\end{tabular}

TABLE IV

The Extended Fixed-Effect Multilevel Linear Regression Model Predicting Three Dependent Variables

\begin{tabular}{|c|c|c|c|c|c|c|c|c|c|c|}
\hline \multirow[b]{2}{*}{ Parameter } & \multirow[b]{2}{*}{$d f_{n u m}$} & \multicolumn{3}{|c|}{ Positive Affect } & \multicolumn{3}{|c|}{ Negative Affect } & \multicolumn{3}{|c|}{ Enjoyment } \\
\hline & & $d f_{d e n}$ & $F$ & $p$ & $d f_{d e n}$ & $F$ & $p$ & $d f_{d e n}$ & $F$ & $p$ \\
\hline Movie Clip & 11 & 193.163 & 35.925 & .000 & 206.260 & 39.739 & .000 & 171.956 & 39.733 & 0 \\
\hline Frame Rate (FR) & 2 & 1071.695 & .180 & .835 & 1045.660 & .478 & 620 & 1136.577 & 4.695 & 0.009 \\
\hline Frame Size (FS) & 1 & 1074.152 & .536 & .464 & 1061.874 & 2.100 & .148 & 1151.402 & 3.336 & 0.068 \\
\hline Bit-Rate (BR) & 1 & 1083.535 & 2.334 & .127 & 1044.851 & .060 & .807 & 1145.171 & 0.257 & 0.612 \\
\hline Extraversion & 1 & 1074.324 & 4.559 & .033 & 1059.767 & .080 & .777 & 1150.401 & 0.024 & 0.877 \\
\hline Agreeableness & 1 & 1072.223 & 1.876 & .171 & 1059.481 & 24.314 & .000 & 1152.475 & 2.001 & 0.157 \\
\hline Conscientiousness & 1 & 1077.950 & 9.474 & .002 & 1041.655 & 3.964 & .047 & 1141.249 & 5.271 & 0.022 \\
\hline Neuroticism & 1 & 1084.026 & .020 & .888 & 1050.845 & 25.227 & .000 & 1146.479 & 0.05 & 0.823 \\
\hline Openness & 1 & 1074.213 & 2.670 & .103 & 1058.628 & 2.110 & .147 & 1145.365 & 4.344 & 0.037 \\
\hline Power Distance & 1 & 1073.888 & 4.676 & .031 & 1055.500 & .000 & .985 & 1152.465 & 9.138 & 0.003 \\
\hline Individualism & 1 & 1070.708 & 2.148 & .143 & 1052.462 & 2.486 & .115 & 1150.026 & 0.674 & 0.412 \\
\hline Masculinity & 1 & 1074.304 & 4.874 & .027 & 1043.258 & 1.061 & .303 & 1141.312 & 3.312 & 0.069 \\
\hline Uncertainty Avoidance & 1 & 1077.284 & .534 & .465 & 1044.360 & .306 & .580 & 1144.106 & 5.751 & 0.017 \\
\hline Pragmatism & 1 & 1069.661 & .886 & .347 & 1064.578 & .175 & .676 & 1160.7 & 0.604 & 0.437 \\
\hline Indulgence & 1 & 1070.162 & 5.863 & .016 & 1051.545 & 4.863 & .028 & 1149.178 & 2.206 & 0.138 \\
\hline
\end{tabular}

had the largest impact on experience of affect and enjoyment. However, it is interesting to note that only Frame Rate had a statistically significant effect on enjoyment. This shows that system factors alone do not make a huge impact on how the content is perceived. That is, given two videos of different 'natures' at different bit-rate, Frame-Rate and Frame-Size, the 'nature' of the content alone is more likely to influence how it is perceived than the system settings at which it is delivered. Our findings can be corroborated by similar observations in quality of experience [37], [38].

b) Extended Model: The extended model adds additional fixed parameters to the baseline model. These were cultural traits including: power distance; individualism; masculinity; uncertainty avoidance; pragmatism; and indulgence. Additionally, personality traits were also added, including: extroversion; agreeableness; conscientiousness; neuroticism; and openness. These were incorporated into the model as covariates with direct effects.

Table IV shows the results of the extended model. It can be seen that several of personality and cultural traits are statistically significant predictors of experience of affect and enjoyment. Among personality traits, extraversion and conscientiousness are significant predictors for positive affect, and agreeableness, neuroticism and conscientiousness are significant for negative affect. Conscientiousness and openness are significant predictors for enjoyment [39], [40]. Among cultural traits, masculinity and indulgence a significant predictor for positive affect, indulgence alone for negative affect and uncertainity avoidance for enjoyment. None of the system factors (except Frame-Rate for enjoyment) and their interactions are significant predictors.

This suggests that the multimedia system characteristics have little to no influence on the intensity of the affect that viewers experience. Additionally, there appears to be a different set of predictors for affect compared to overall enjoyment. It is important to note that the $F$-statitic is generally quite small for most of the predictors. However, the predictors of agreeableness and neuroticism, for negative affect, are notably much larger. This suggests that a considerable amount of the variance in negative affect can be explained by these parameters.

c) Optimistic Model: While the goal of a model is to predict the value of a dependent variable as accurately as possible, not all of the residual variance can be solely attributed to human factors which have not been measured. A non-trivial proportion of the residual variance can also, to 
TABLE V

An Optimistic MiXed-Effect Multilevel Linear Regression Model Predicting Three Dependent Variables

\begin{tabular}{|c|c|c|c|c|c|c|c|c|c|c|}
\hline \multirow[b]{2}{*}{ Parameter } & \multirow[b]{2}{*}{$d f_{n u m}$} & \multicolumn{3}{|c|}{ Positive Affect } & \multicolumn{3}{|c|}{ Negative Affect } & \multicolumn{3}{|c|}{ Enjoyment } \\
\hline & & $d f_{d e n}$ & $F$ & $p$ & $d f_{d e n}$ & $F$ & $p$ & $d f_{d e n}$ & $F$ & $p$ \\
\hline Movie Clip & 11 & 178.713 & 42.312 & .000 & 152.624 & 55.782 & .000 & 179.877 & 46.99 & .000 \\
\hline Frame Rate & 2 & 701.036 & 1.788 & .168 & 945. & 1.392 & .249 & 1116.89 & 8.025 & .000 \\
\hline Frame Size (FS) & 1 & 695.825 & .002 & .965 & 969.366 & 5.764 & .017 & 1120.818 & 3.13 & 0.077 \\
\hline Bit-Rate (BR) & 1 & 715.664 & 1.159 & .282 & 972.050 & 1.457 & .228 & 1121.96 & 0.054 & 0.816 \\
\hline
\end{tabular}

name a few, be attributed to: random error; measurement error; and the limitations of the modelling technique (in this case, generalised linear regression). As such, an optimistic model can be used to estimate the proportion of this residual variance which can be reasonably attributed to human factors (and, to a small extent because of limitations to experimental control, context factors). This is achieved by modelling each participant as a "random effect". That is, the repeated measurements were used to vary the intercept of the regression for each individual participant.

Results from the optimistic models are shown in Table $\mathrm{V}$. There is only a small number of differences between the baseline and the optimistic model. The F-statistics for the intercepts are much larger, showing that they explain a larger proportion of the variance. Additionally, the borderline significant interaction between Frame-Size and experience of affect has become significant. The most notable difference, however, is a large increase in the variance explained as a result of including participants as random effects.

\section{B. Model Comparison}

The models are compared using paired t-tests on the Mean Squared Residuals (MSR), shown in Table VI, and the proportional reduction in overall mean squared error of prediction is examined (see [41]). The results show that human factors namely personality and culture play a crucial role in modeling the experience of affect and enjoyment, indicating that content production and delivery mechanisms should not just take into account multimedia system factors but also human factors to achieve maximal user satisfaction.

1) Models for Positive Emotion: In the baseline model, the MSR is $0.6304(\sigma=1.050)$. The optimistic model reduces the MSR to $0.4051(\sigma=0.886)(p<.000)$. This represents $55.3 \%$ of the overall variance predicted, a part of which is contributed by culture and personality. The extended model predicts approximately $5.6 \%$ of variance attributable to human factors, reducing the baseline MSR to 0.6177 ( $\sigma=1.005)$ $(p=.021)$.

2) Models for Negative Emotion: In the baseline model, the MSR is $0.6514(\sigma=0.889)$. The optimistic model reduces the MSR to $0.3615(\sigma=0.536)(p<.000)$. This represents $58.1 \%$ of the overall variance predicted. The extended model predicts approximately $13.6 \%$ of variance attributable to human factors, reducing the baseline MSR to $0.6118(\sigma=0.8278)(p<.000)$.
3) Models for Enjoyment: In the baseline model, the MSR is $1.3684(\sigma=1.63)$. The optimistic model reduces the MSR to $0.9481(\sigma=1.22)(p<.000)$. This represents $23.0 \%$ of the overall variance predicted (compared to $47.8 \%$ overall). The extended model predicts approximately $9.3 \%$ of variance attributable to human factors, reducing the baseline MSR to $1.3290(\sigma=1.58)(p<.001)$.

\section{Correlation b/w Affect and Enjoyment}

As introduced at the beginning of the article, there is a very close and significant relationship between what users enjoy and the emotion it evokes (results from correlation analysis are shown in Table VII). In all clips, enjoyment is significantly correlated with interest, joy, satisfaction and the latent factor, positive emotion. This means that for a user to enjoy a video the content has to no doubt draw his/her interest but also have moments of happiness and must deliver something which satisfies the person [42].

There are also very few instances of negative emotions (sad, fearful, guilty and ashamed) giving enjoyment to users. These might be associated with how certain users (possibly with high scores on neuroticism) perceive certain contents [43], [44].

Apart from that, even the nature of the content itself can arouse contradictory emotions. For example, enjoyment is observed to be positively correlated with sadness in the movie clip FOREST_GUMP which has a very unique sequence where the protagonist of the movie sees his son for the first time and leaves the audience both in sadness and joy, due to the fact that he realises that he has a son after such a long time and that his son is doing well in school and is a fine student. So, such occurrences are due to the interplay between both human factors and nature of the content.

It is interesting to note that while most of the users might associate enjoyment with positivity, there are certain users who need to experience negative emotions to connect to the content's message. This insight gives content creators a better understanding of how to influence users with different personality and cultural traits to establish an emotional connection with them, which is very important to drive behavioral action (especially in scenarios involving ad campaign design etc.).

\section{CONClusion}

Experience of affect and enjoyment in multimedia is influenced by an intricate interplay between characteristics of stimuli, individuals, and systems of perception. Knowing the 
TABLE VI

PAIRED T-TEST COMPARING MODELS FOR ALL THREE RESPONSES (W.R.T MSR)

\begin{tabular}{|c|c|c|c|c|c|c|c|c|c|c|c|c|}
\hline \multirow[b]{2}{*}{ Models } & \multicolumn{4}{|c|}{ Positive Affect } & \multicolumn{4}{|c|}{ Negative Affect } & \multicolumn{4}{|c|}{ Enjoyment } \\
\hline & $\Delta \bar{x}$ & $\sigma$ & $t$ & $p$ & $\Delta \bar{x}$ & $\sigma$ & $t$ & $p$ & $\Delta \bar{x}$ & $\sigma$ & $t$ & $\bar{p}$ \\
\hline Baseline $\rightarrow$ Extended & .0127 & .193 & 2.311 & .021 & .0396 & .277 & 5.008 & .000 & .0394 & .430 & 3.219 & .001 \\
\hline Baseline $\rightarrow$ Optimistic & .2253 & .924 & 8.552 & .000 & .2898 & .726 & 14.014 & .000 & .4199 & 1.129 & 13.069 & .000 \\
\hline
\end{tabular}

TABLE VII

SigNifiCANT CORRELATIONS BETWEEN ENJOYMENT AND EXPERIENCE OF AFFECT $(p<0.05)$

\begin{tabular}{l|rrrrrrrrrrrrrr}
\hline Clip & Interest & Joy & Sad & Fearful & Disgust & Surprise & Warm & Loving & Guilty & Moved & Satisfied & Calm & Ashamed + +ve Affect \\
\hline C-I & .579 & .699 & - & - & -.332 & .430 & .626 & .419 & - & - & .456 & .451 & - & .610 \\
C-II & .505 & .304 & - & - & -.332 & .380 & .350 & .383 & .258 & - & .526 & .404 & .291 & .483 \\
C-III & .596 & .485 & - & - & -.314 & .250 & .298 & .247 & - & - & .432 & .286 & - & .426 \\
C-IV & .444 & .424 & - & - & - & - & .287 & .243 & - & - & .255 & - & - & .304 \\
C-V & .469 & .263 & $-j u s t$ & - & - & - & .368 & .271 & .244 & .219 & .288 & .232 & .250 & .298 \\
C-VI & .514 & .385 & - & -.239 & - & - & .353 & .325 & - & - & .493 & - & -.215 & .456 \\
C-VII & .549 & .643 & .248 & - & - & .293 & .479 & .513 & - & .508 & .510 & .407 & - & .560 \\
C-VIII & .550 & .408 & - & - & - & - & - & - & - & .346 & .445 & .319 & - & .421 \\
C-IX & .340 & .394 & - & - & - & - & .323 & .251 & - & - & .219 & .244 & - & .292 \\
C-X & .512 & .658 & - & - & - & .258 & .301 & - & - & - & .267 & .354 & .290 & .419 \\
C-XI & .541 & .266 & - & - & - & .232 & - & - & - & .277 & .358 & .325 & - & .347 \\
C-XII & .590 & .688 & - & - & -.401 & .317 & .434 & .419 & .244 & .295 & .488 & .353 & - & .542 \\
\hline
\end{tabular}

Movie Clips:- C-I: A_FISH_CALLED_WANDA; C-II: AMERICAN_HISTORY_X; C-III: CHILDS_PLAY_II; C-IV:COPYCAT; C-V: DEAD_POETS_SOCIETY_I; C-VI: DEAD_POETS_SOCIETY_2; C-VII: FOREST_GUMP; C-VIII: SE7EN_I; C-IX: SETEN_3; C-X: SOMETHING_ABOUT_MARY; C-XI: THE_PROFESSIONAL; C-XII: TRAINSPOTTING. No significant correlations were observed between Enjoyment and Anxious, Angry, Disdain \& -ve Affect and thus are not shown in the table. Entry $E_{i, j}$ represents the correlation between enjoyment and affect for movie clip $i$ and emotion category $j$

impact of these factors permits them to be exploited to optimise enjoyment under conditions where content delivery is constrained.

Results indicate that human factors play an important role in the way users experience affect and enjoyment. It is important to recognise that the human factors explored in this study, namely personality and culture, represent a small portion of the variance which can be attributed to human factors overall. Nonetheelss, results show that human factors namely personality and culture play a significant role in modeling the experience of affect and enjoyment, indicating that content production and delivery mechanisms should not just take into account multimedia system factors but also human factors to achieve maximal user satisfaction.

Specifically, the conclusions of this work towards the research questions raised in this paper are as follows:

RQ1 For positive affect, negative affect and enjoyment, personality and culture represented $5.6 \%, 13.6 \%$ and $9.3 \%$ of the variance respectively. While this is an important proportion, further study is needed to discover other contributing factors, which could include sensory impairments and expertise.

RQ2 Traits of extraversion, conscientiousness, masculinity and indulgence are significant predictors for positive affect, and agreeableness, neuroticism, conscientiousness and indulgence were important predictors for negative affect, and conscientiousness, openness and uncertainity avoidance were signifi- cant predictors for enjoyment.

RQ3 The majority of the movie clips which were enjoyed were also rated high on positive affect, with a small exception of clips having high correlation between negative affect and enjoyment. Such behavior is possibly due to the interplay between human factors (like neuroticism) and nature of the content.

Further work is suggested on developing systems which can automatically detect the experience of affect and enjoyment given the content taking into consideration the variance in personality and cultural traits.

\section{REFERENCES}

[1] "Dove, unilever," Accessed: 2015-04-20.

[2] Jacquelyn Smith, "The most unforgettable ad campaigns of 2013," December 2013.

[3] Peter Noel Murray, "How emotions influence what we buy," February 2013.

[4] Susan T Fiske, Amy JC Cuddy, and Peter Glick, "Universal dimensions of social cognition: Warmth and competence," Trends in cognitive sciences, vol. 11, no. 2, pp. 77-83, 2007.

[5] Kathy A Winter and Nicholas A Kuiper, "Individual differences in the experience of emotions," Clinical psychology review, vol. 17, no. 7, pp. 791-821, 1997.

[6] Wonhee Choe, Hyo-Sun Chun, Junhyug Noh, Seong-Deok Lee, and Byoung-Tak Zhang, "Estimating multiple evoked emotions from videos," in Proceedings of Annual Meeting of the Cognitive Science Society, 2013, pp. 2046-2051.

[7] Gerald Matthews, Ian J Deary, and Martha C Whiteman, Personality traits, Cambridge University Press, 2003.

[8] Geert Hoftede, Gert Jan Hofstede, and Michael Minkov, Cultures and organizations: software of the mind: intercultural cooperation and its importance for survival, McGraw-Hill, 2010. 
[9] Zhihong Zeng, Maja Pantic, Glenn I Roisman, and Thomas S Huang, "A survey of affect recognition methods: Audio, visual, and spontaneous expressions," Pattern Analysis and Machine Intelligence, IEEE Transactions on, vol. 31, no. 1, pp. 39-58, 2009.

[10] Luca Canini, Sergio Benini, and Riccardo Leonardi, "Affective recommendation of movies based on selected connotative features," Circuits and Systems for Video Technology, IEEE Transactions on, vol. 23, no. 4, pp. 636-647, 2013.

[11] Subhabrata Bhattacharya, Behnaz Nojavanasghari, Tao Chen, Dong Liu, Shih-Fu Chang, and Mubarak Shah, "Towards a comprehensive computational model foraesthetic assessment of videos," in Proceedings of the 21st ACM international conference on Multimedia. ACM, 2013, pp. 361-364.

[12] Cheng-Yu Wei, Nevenka Dimitrova, and Shih-Fu Chang, "Colormood analysis of films based on syntactic and psychological models," in Multimedia and Expo, 2004. ICME'04. 2004 IEEE International Conference on. IEEE, 2004, vol. 2, pp. 831-834.

[13] Sergio Benini, Luca Canini, and Riccardo Leonardi, "A connotative space for supporting movie affective recommendation," Multimedia, IEEE Transactions on, vol. 13, no. 6, pp. 1356-1370, 2011.

[14] Björn Schuller, Michel Valstar, Florian Eyben, Gary McKeown, Roddy Cowie, and Maja Pantic, "Avec 2011-the first international audio/visual emotion challenge," in Affective Computing and Intelligent Interaction, pp. 415-424. Springer, 2011.

[15] Rajitha Navarathna, Patrick Lucey, Peter Carr, Elizabeth Carter, Sridha Sridharan, and Iain Matthews, "Predicting movie ratings from audience behaviors," in Applications of Computer Vision (WACV), 2014 IEEE Winter Conference on. IEEE, 2014, pp. 1058-1065.

[16] Mohammad Soleymani, Maja Pantic, and Thierry Pun, "Multimodal emotion recognition in response to videos," Affective Computing, IEEE Transactions on, vol. 3, no. 2, pp. 211-223, 2012.

[17] Sander Koelstra, Christian Muhl, Mohammad Soleymani, Jong-Seok Lee, Ashkan Yazdani, Touradj Ebrahimi, Thierry Pun, Anton Nijholt, and Ioannis Patras, "Deap: A database for emotion analysis; using physiological signals," Affective Computing, IEEE Transactions on, vol. 3 , no. 1, pp. 18-31, 2012.

[18] Yoann Baveye, J-N Bettinelli, Emmanuel Dellandréa, Liming Chen, and Christel Chamaret, "A large video database for computational models of induced emotion," in Affective Computing and Intelligent Interaction (ACII), 2013 Humaine Association Conference on. IEEE, 2013, pp. 1318.

[19] Yu-Gang Jiang, Baohan Xu, and Xiangyang Xue, "Predicting emotions in user-generated videos," in Twenty-Eighth AAAI Conference on Artificial Intelligence, 2014.

[20] Jussi Tarvainen, Mats Sjoberg, Stina Westman, Jorma Laaksonen, and Pirkko Oittinen, "Content-based prediction of movie style, aesthetics and affect: Data set and baseline experiments," 2014.

[21] Julien Fleureau, Philippe Guillotel, and Izabela Orlac, "Affective benchmarking of movies based on the physiological responses of a real audience," in Affective Computing and Intelligent Interaction (ACII), 2013 Humaine Association Conference on. IEEE, 2013, pp. 73-78.

[22] Alexandre Schaefer, Frédéric Nils, Xavier Sanchez, and Pierre Philippot, "Assessing the effectiveness of a large database of emotion-eliciting films: A new tool for emotion researchers," Cognition and Emotion, vol. 24 , no. 7, pp. 1153-1172, 2010.

[23] Mojtaba Khomami Abadi, Seyed Mostafa Kia, Ramanathan Subramanian, Paolo Avesani, and Nicu Sebe, "User-centric affective video tagging from meg and peripheral physiological responses," in Affective Computing and Intelligent Interaction (ACII), 2013 Humaine Association Conference on. IEEE, 2013, pp. 582-587.

[24] Julia Wache, "The secret language of our body: Affect and personality recognition using physiological signals," in Proceedings of the 16th International Conference on Multimodal Interaction. ACM, 2014, pp. 389-393.

[25] Geert Hofstede, "Dimensionalizing cultures: The hofstede model in context," Online readings in psychology and culture, vol. 2, no. 1, pp. 8, 2011.

[26] Shlomo Argamon, Sushant Dhawle, Moshe Koppel, and James Pennebaker, "Lexical predictors of personality type," 2005.

[27] Gelareh Mohammadi and Alessandro Vinciarelli, "Humans as feature extractors: combining prosody and personality perception for improved speaking style recognition," in Systems, Man, and Cybernetics (SMC), 2011 IEEE International Conference on. IEEE, 2011, pp. 363-366.
[28] Sharath Chandra Guntuku, Sujoy Roy, and Weisi Lin, "Personality modeling based image recommendation," in MultiMedia Modeling. Springer, 2015, pp. 171-182.

[29] Jennifer Golbeck, Cristina Robles, and Karen Turner, "Predicting personality with social media," in CHI'11 extended abstracts on human factors in computing systems. ACM, 2011, pp. 253-262.

[30] Alessandro Vinciarelli and Gelareh Mohammadi, "A survey of personality computing," 2014.

[31] Saham Barza and Mehran Memari, "Movie genre preference and culture," Procedia-Social and Behavioral Sciences, vol. 98, pp. 363368, 2014.

[32] C Samuel Craig, William H Greene, and Susan P Douglas, "Culture matters: consumer acceptance of us films in foreign markets," Journal of International Marketing, vol. 13, no. 4, pp. 80-103, 2005.

[33] Geert Hofstede, Gert Jan Hofstede, Michael Minkov, and Henk Vinken, "Values survey module 2013," URL: http://www.geerthofstede.nl/vsm2013, 2013.

[34] Samuel D Gosling, Peter J Rentfrow, and William B Swann, "A very brief measure of the big-five personality domains," Journal of Research in personality, vol. 37, no. 6, pp. 504-528, 2003.

[35] Gregory J McHugo, Craig A Smith, and John T Lanzetta, "The structure of self-reports of emotional responses to film segments," Motivation and Emotion, vol. 6, no. 4, pp. 365-385, 1982.

[36] Lewis R Goldberg, "An alternative" description of personality": the bigfive factor structure.," Journal of personality and social psychology, vol. 59, no. 6, pp. $1216,1990$.

[37] George Ghinea and Johnson P Thomas, "Qos impact on user perception and understanding of multimedia video clips," in Proceedings of the sixth ACM international conference on Multimedia. ACM, 1998, pp. 49-54.

[38] Nick Yeung and Alan G Sanfey, "Independent coding of reward magnitude and valence in the human brain," The Journal of Neuroscience, vol. 24, no. 28, pp. 6258-6264, 2004.

[39] Luiz Pessoa, "On the relationship between emotion and cognition," Nature Reviews Neuroscience, vol. 9, no. 2, pp. 148-158, 2008.

[40] Carroll E Izard, Deborah Z Libero, Priscilla Putnam, and O Maurice Haynes, "Stability of emotion experiences and their relations to traits of personality.," Journal of personality and social psychology, vol. 64, no. 5 , pp. 847,1993

[41] Roel Bosker and Tom Snijders, "Multilevel analysis: An introduction to basic and advanced multilevel modeling, 2nd ed," New York, 2012.

[42] Maria Teresa Soto Sanfiel, Laura Aymerich Franch, F Xavier Ribes Guardia, and J Reinaldo Martinez Fernandez, "Influence of interactivity on emotions and enjoyment during consumption of audiovisual fictions," International Journal of Arts and Technology, vol. 4, no. 1, pp. 111-129, 2011.

[43] Randy J Larsen and Timothy Ketelaar, "Personality and susceptibility to positive and negative emotional states.," Journal of personality and social psychology, vol. 61, no. 1, pp. 132, 1991.

[44] Ed Diener, Shigehiro Oishi, and Richard E Lucas, "Personality, culture, and subjective well-being: Emotional and cognitive evaluations of life," Annual review of psychology, vol. 54, no. 1, pp. 403-425, 2003. 